\title{
Quantum Field Kinetics
}

\author{
A. Makhlin \\ Department of Physics and Astronomy, Wayne State University, Detroit, MI 48202
}

(June 19, 2021)

\begin{abstract}
Using the general framework of quantum field theory, we derive basic equations of quantum field kinetics. The main goal of this approach is to compute the observables associated with a quarkgluon plasma at different stages of its evolution. We start by rewriting the integral equations for the field correlators in different forms, depending on the relevant dynamical features at each different stage. Next, two versions of perturbation expansion are considered. The first is best suited for the calculation of electromagnetic emission from chaotic, but not equilibrated, strongly interacting matter. The second version allows one to derive evolution equations, which are generalizations of the familiar QCD evolution equations, and provide a basis for the calculation of the initial quark and gluon distributions after the first hard interaction of the heavy ions.
\end{abstract}

12.38.Mh, 12.38.Bx, 25.75.+r

\section{Introduction}

The most ambitious goal of the RHIC and LHC programs is to discover a new state of the matter - the quark-gluon plasma (QGP). Evidence for a QGP will require a self-consistent analysis of many signals from all stages of the collision - initial "hard" processes $(\tau<1 / 2 \mathrm{fm} / \mathrm{c})$, the QGP itself, and from the "cool" hadronic gas $(\tau \sim 10-30 \mathrm{fm} / \mathrm{c})$. Thus, a continuous description for all $\tau$ is of more than of academic interest. This is a difficult task, and currently, each stage is described using different approach. Here, we primarily wish to design a formalism that allows one to describe all stages of the collision, including the transient ones, using the same technical tools.

The existence of a QGP is inseparable from the process of creation of the matter it consists of. It is an essentially quantum process . Therefore an exact definition of the initial state of the system, and of the observables in the expected final state, is required. Two difficulties arise: (i) It is unclear how the stable nuclei of the initial state are build up from quarks and gluons; and (ii) the expected final state is imperfectly understood. Thus, the theory should be adaptable enough to deal with these uncertainties. It must bridge the logical gap between the language used to describe stable nuclei and a QGP, as is done phenomenologically in using an intermediate free parton language. More specifically, in describing a transition between the initial state of two stable, well shaped nuclei, and a system of free quarks and gluons, we encounter one of the most painful problems of quantum field theory - initial and final states of the system can not be described in terms of the same language. Before the initial collision, the system is confined and the vacuum is dominated by quark and gluon condensates, while afterwards all the condensates are destroyed and the quark-gluon dynamics is calculated with respect to the perturbative vacuum. This vacuum itself is a product of the collision.

We may begin the study with the assumption that a high multiplicity of quarks and gluons has already been created. However, this "plasma" may remain out of thermal and chemical equilibrium for a long time, and may not reach it at all 37. In this case, the calculation of even relatively simple signatures such as dilepton and photon emission is a nontrivial problem. An overview of the many publications on this subject reveals that the QGP has generally been treated as a totally equilibrated system, and that calculations are essentially based on the detail balance relationships.

Therefore, we may conclude that any reasonable theory should rely neither on detail balance and thermal equilibrium, nor even on the existence of the same ground state for all stages of the QGP evolution. The theory should explicitly follow the temporal sequence of the stages, and allow for smooth transitions between them. The prototype of such a theory was designed by Keldysh [1] for non-equilibrium condensed matter systems with the aim of deriving a quasi-classical kinetic equation.

The version of the Keldysh technique presented below, and named "Quantum Field Kinetics" (QFK), takes an intermediate position between the theory of scattering and quantum field theory of the many-particle systems. It contains both as its limits. On the one hand, it allows one to calculate the inclusive cross-sections and rates of emission since the summation over all unobserved states is implicit. Also, it provides the proper balance between "real processes" and "radiative corrections" which results in the cancellation of infrared divergencies, and makes unnecessary to introduce artificial intermediate cut-offs. On the other hand, QFK allows one to calculate local observables for many-particle systems. As a by-product it recovers microcausality, precisely in the form which was used in axiomatic field theory.

For any particular problem, QFK-based calculations always begin with the basic definition of observables in terms of their Heisenberg operators, the Lagrangian of the theory, and the density matrix of the initial state. Several examples, motivated by the expected scenario in heavy ion collisions and possible probes of the QGP, are described in Section 2. One may express the observables via field correlators with differently ordered field operators. The correlators are 
naturally arrayed in $2 \times 2$ matrices, and we follow the original Keldysh idea of contour ordering. The correlators obey a system of matrix equations of the Schwinger-Dyson type, which is easily derived via functional methods. The equations are reviewed in Sec.3.3.

Starting with the initial form of the matrix equations, it is straightforward to generate a formal perturbation series for the probabilities of inclusive processes (instead of amplitubes for exclusive ones) in powers of the coupling constant. This series reproduces the Feynman expansion diagram for diagram, except that now each vertex acquires an additional dichotomic index. The way to sum this perturbation series is always influenced by the physically motivated renormalization conditions, which may be different from case to case.

As a preliminary step for the future rearrangement of the perturbation series, we begin, also following Keldysh, with a rotation of the matrix basis. We introduce the retarded and advanced Green functions of QCD along with additional correlators which carry information about the space of states and derive the equations they obey. In Section 3.4 we show that the latter allow for a formal solution which comes to be the first approximation of the QCD evolution equations, if we renormalize the retarded propagators according to the requirement of light cone propagation.

It is important to note that instead of using the traditional method of the operator product expansion, we can derive the evolution equations for the DIS structure functions using the language of the QFK. This language avoids the parton phenomenology which is needed when DIS structure functions are used for the computation of the quark and gluon distributions in first hard collision of the two nuclei. Thus, QFK provides a firmer footing for the description of heavy ion collisions.

In Section 4 we discuss different versions of the perturbation expansion. We show that the ordinary perturbation series in powers of the coupling constant is adequate for calculations of systems with slowly varying macroscopic parameters. We describe the scheme using the example of dilepton emission from the QGP. An expansion which is suitable for the violent impact of relativistic composite systems requires another kind of expansion, one which preserves the leading light cone singularity of the propagators.

\section{Signals from the quark-gluon plasma}

In this section we begin the design of a technique which will allow us to calculate different observables associated with QGP. The latter should be defined unambiguously at both the theoretical and apparatus level. We shall try to trace their origin using only the basic principles of quantum mechanics for as long as is possible. If successful, we may then express the signals in terms of the parameters of the emitting system. All the systems we shall study are described by the standard QCD Lagrangian. In order to deal with electromagnetic probes we include the interaction of charged quarks, $q(x)$, and leptons, $\psi(x)$, with the photon field, $A^{\mu}(x)$, in the total renormalized interaction Lagrangian:

$$
\begin{array}{r}
\mathcal{L}_{i n t}(x)=e \bar{\psi}(x) \gamma^{\mu} \psi(x) A_{\mu}(x)+e \sum_{q, i} \bar{q}_{i}(x) \gamma^{\mu} A_{\mu}(x) q_{i}(x)+g_{r} \sum_{q, i} \bar{q}_{i}(x) t_{i j}^{a} \gamma^{\mu} B_{\mu}^{a}(x) q_{j}(x)+ \\
+g_{r} f_{a b c} \partial^{\mu} B_{a}^{\nu}(x) B_{\mu}^{b}(x) B_{\nu}^{c}(x)+\left(g_{r}^{2} / 4\right) f_{a b c} f_{a g h} B_{b}^{\mu}(x) B_{c}^{\nu}(x) B_{\mu}^{g}(x) B_{\nu}^{h}(x),
\end{array}
$$

where $B_{a}^{\mu}(x)$ is the gluon field, and $g_{r}$ is a renormalized strong coupling constant. Other notation is commonly used, and requires no comment. The standard counterterms which will be used for renormalization are as follows:

$$
\begin{array}{r}
\mathcal{L}_{C T}(x)=\frac{Z_{3}-1}{2} B_{a}^{\mu}\left(g_{\mu \nu} \partial^{2}-\partial_{\mu} \partial_{\nu}+\lambda n_{\mu} n_{\nu}\right) B_{a}^{\nu}-\left(Z_{1}-1\right) g_{r} f_{a b c} \partial^{\mu} B_{a}^{\nu}(x) B_{\mu}^{b}(x) B_{\nu}^{c}(x)- \\
-\left(Z_{4}-1\right) \frac{g_{r}^{2}}{4} f_{a b c} f_{a g h} B_{b}^{\mu}(x) B_{c}^{\nu}(x) B_{\mu}^{g}(x) B_{\nu}^{h}(x)+\left(Z_{2}-1\right) \bar{q}_{i}(x) i \gamma^{\mu} \partial_{\mu} q_{i}(x)+\left(Z_{1 F}-1\right) g_{r} \bar{q}_{i}(x) t_{i j}^{a} \gamma^{\mu} B_{\mu}^{a}(x) q_{j}(x)
\end{array}
$$

Here, all $(Z-1)$ 's are considered as the small parameters. In what follows we shall either use covariant gauges in the lowest order calculations when the ghosts do not contribute yet, or shall work in the axial gauge, $B^{\mu} n_{\mu}=0$, where they are absent.

The Lagrangian (2.1)- (2.2) gives rise to the ordinary S-matrix in the in-interaction picture,

$$
S=T \exp \left\{i \int d^{4} x \mathcal{L}_{\text {int }}(x)\right\}
$$

which is considered to be a limit of the evolution operator that governs the dynamics of Heisenberg observables.

The initial state for any system can be described by the density matrix $\rho_{Q C D}$. It is formed due to strong interactions only, and we shall specify it later. When the inclusive cross-sections of photon and dilepton emission are chosen as the observables, this density operator should be augmented by the projector on the initial vacuum state of photons and leptons, 


$$
\rho=\rho_{Q C D} \otimes\left|0_{e \gamma}\right\rangle\left\langle 0_{e \gamma}\right|
$$

and we assume that the QGP remains transparent for photons and leptons throughout its history. The amplitudes of the transition from one initial state, $|i n\rangle$, to a final one containing photon or dilepton read as

$$
\langle X|c(\mathbf{k}, \lambda) S| i n\rangle \quad \text { or } \quad\langle X|b(2) a(1) S| i n\rangle \quad,
$$

where $c(\mathbf{k}, \lambda)$ and $a(J)=a\left(\mathbf{k}_{J}, \sigma_{J}\right)$ and $b(J)$ are the photon, electron and positron annihilation operators. Summing the squared moduli of these amplitudes over a complete set of uncontrolled states $|X\rangle$, and averaging over the initial ensemble, we find the inclusive spectra of photons and dileptons,

$$
\begin{gathered}
\frac{d N_{\gamma}}{d \mathbf{k}}=\sum_{\lambda} \operatorname{Sp} \rho_{i n} S^{\dagger} c^{\dagger}(\mathbf{k}, \lambda) c(\mathbf{k}, \lambda) S, \\
\frac{d N_{e^{+} e^{-}}}{d \mathbf{k}_{1} d \mathbf{k}_{2}}=\sum_{\sigma_{1}, \sigma_{2}} \operatorname{Sp} \rho_{i n} S^{\dagger} a^{\dagger}(1) b^{\dagger}(2) b(2) a(1) S .
\end{gathered}
$$

It is easy now to commute the Fock operators with $S$ and $S^{\dagger}$. Due to the QED vacuum projector in the density matrix (2.4), only the commutators survive and Eq.(2.6) takes the form:

$$
k^{0} \frac{d N_{\gamma}}{d \mathbf{k} d^{4} x}=\frac{i g_{\mu \nu}}{2(2 \pi)^{3}} \pi_{10}^{\mu \nu}(-k),
$$

where $k^{0}>0$, and the polarization tensor

$$
\pi_{10}^{\mu \nu}(-k)=-i \int d^{4}(x-y) e^{-i k(x-y)}\left\langle\frac{\delta S^{\dagger}}{\delta A^{\mu}(x)} \frac{\delta S}{\delta A^{\nu}(y)}\right\rangle,
$$

is the Fourier transform of the product of two Heisenberg electromagnetic currents averaged with the density matrix $\rho_{Q C D}$ :

$$
\pi_{10}^{\mu \nu}(x, y)=i\left\langle\frac{\delta S^{\dagger}}{\delta A^{\mu}(x)} \frac{\delta S}{\delta A^{\nu}(y)}\right\rangle=i\left\langle\mathbf{j}^{\mu}(x) \mathbf{j}^{\nu}(y)\right\rangle
$$

The Heisenberg current (as any other local Heisenberg operator) can be written down in the two equivalent ways,

$$
\mathbf{j}^{\mu}(x)=S^{\dagger} T\left(j^{\mu}(x) S\right) \equiv T^{\dagger}\left(j^{\mu}(x) S^{\dagger}\right) S,
$$

where $j^{\mu}(x)=(1 / 2) \sum_{i, q} e_{q}\left[\bar{q}_{i}(x) \gamma^{\mu}, q_{i}(x)\right]$, is the operator of the electromagnetic current in the in-interaction picture. Relations such as (2.11) are extremely important as they allow one to keep the initial order of the operators through all stages of calculations. This order is strictly prescribed by the definition of the observables and may not be changed safely, except under very special circumstances.

Now the dilepton rate of emission (2.7) takes the form

$$
k_{1}^{0} k_{2}^{0} \frac{d N_{e^{+} e^{-}}}{d \mathbf{k}_{1} d \mathbf{k}_{2} d^{4} x}=-i e^{2} \frac{L_{\mu \nu}\left(k_{1}, k_{2}\right)}{4(2 \pi)^{6}} \boldsymbol{\Delta}_{10}^{\mu \nu}(-k),
$$

where $k=k_{1}+k_{2}$, and $L^{\mu \nu}=k_{1}^{\mu} k_{2}^{\nu}+k_{2}^{\mu} k_{1}^{\nu}-g^{\mu \nu}\left(k_{1} k_{2}-m_{e}^{2}\right)$ is the polarization sum of the lepton spinors. The electromagnetic correlator

$$
\boldsymbol{\Delta}_{10}^{\mu \nu}(-k)=-i \int d^{4}(x-y)\left\langle T^{\dagger}\left(A^{\mu}(x) S^{\dagger}\right) T\left(A^{\mu}(y) S\right)\right\rangle e^{-i k(x-y)}
$$

is a kind of photon Wightman function averaged over $\rho^{Q C D}$. The operator $A(x)$ of the in-interaction picture and the Heisenberg operator $\mathbf{A}(x)$ are connected via relations similar to (2.11). In the absence of radiative corrections to the photon propagation, dynamical equations which will be derived in the next Section will allow us to rewrite the photon correlator in Eq. (2.12) as following,

$$
\boldsymbol{\Delta}_{10}^{\mu \nu}(k)=-\Delta_{r e t}(k) \pi_{10}^{\mu \nu}(k) \Delta_{a d v}(k)=-\pi_{10}^{\mu \nu}(k) /\left[k^{2}\right]^{2} .
$$


In order to derive Eqs. (2.8) and (2.12) we have assumed that an explicit separation of long-range and short-range scales is possible, and have introduced the emission rates per unit volume, instead of the inclusive cross-sections.

The way we proceeded above demonstrates the very simple and general principle of formulation of the problem: Observables like cross-sections or the rates of emission must be expressed in terms of specifically ordered products of the Heisenberg operators. If we change the photon and lepton creation and annihilation operators in the Eq. (2.5))(2.7) for those of quarks or gluons, and replace the $\rho_{i n}$ of the QGP by a density matrix for the two colliding nuclei, we obtain the starting point for a computation of the quark and gluon distributions after the first hard collision.

We now require a formalism which will allow us to calculate these quantities. We are to keep in mind that all the operators are driven by $\mathcal{L}_{i n t}$, and that all the information about the initial state of the system is hidden in $\rho_{i n}$. Nothing else is needed to solve the problem.

\section{The equations of relativistic quantum field kinetics}

\subsection{Basic definitions}

In this study, the calculation of observables such as the emission rate is based on work by Keldysh [1]. It incorporates a specific set of exact (dressed) field correlators. These correlators are products of Heisenberg operators, averaged with the density matrix of the initial state. For the quark field they read

$$
\begin{aligned}
& \mathbf{G}_{10}(x, y)=-i\langle\mathbf{q}(x) \overline{\mathbf{q}}(y)\rangle, \quad \mathbf{G}_{01}(x, y)=i\langle\overline{\mathbf{q}}(y) \mathbf{q}(x)\rangle, \\
& \mathbf{G}_{00}(x, y)=-i\langle T(\mathbf{q}(x) \overline{\mathbf{q}}(y))\rangle, \mathbf{G}_{11}(x, y)=-i\left\langle T^{\dagger}(\mathbf{q}(x) \overline{\mathbf{q}}(y))\right\rangle,
\end{aligned}
$$

where $T$ and $T^{\dagger}$ are the symbols of the time and anti-time ordering. They may be rewritten in a unified form,

$$
\mathbf{G}_{A B}(x, y)=-i\left\langle T_{c}\left(\mathbf{q}\left(x_{A}\right) \overline{\mathbf{q}}\left(y_{B}\right)\right)\right\rangle,
$$

in terms of a special ordering $T_{c}$ along a contour $C=C_{0}+C_{1}$, the doubled time axis, with $T$-ordering on $C_{0}$ and $T^{\dagger}$-ordering on $C_{1}$. The operators labelled by ' 1 ' are $T^{\dagger}$-ordered, and stand before the $T$-ordered operators labelled by ' 0 '. Recalling that

$$
\mathbf{q}(x)=S^{\dagger} T(q(x) S) \equiv T^{\dagger}(q(x) S) S,
$$

we may introduce the formal operator $S_{c}=S^{\dagger} S$, and rewrite (3.2) using the operators of the in-interaction picture

$$
\mathbf{G}_{A B}(x, y)=-i\left\langle T_{c}\left(q\left(x_{A}\right) \bar{q}\left(y_{B}\right) S_{c}\right)\right\rangle,
$$

where, by the definition, the internal variables of $S$ lie on $C_{0}$, and those of $S^{\dagger}$ on $C_{1}$.

The boson Greenians are built in the same manner, i.e., for gluon field, $B(x)$, and photon field, $A(x)$, we have

$$
\begin{aligned}
& \mathbf{D}_{A B}(x, y)=-i\left\langle T_{c}\left(B\left(x_{A}\right) B\left(y_{B}\right) S_{c}\right)\right\rangle, \\
& \boldsymbol{\Delta}_{A B}(x, y)=-i\left\langle T_{c}\left(A\left(x_{A}\right) A\left(y_{B}\right) S_{c}\right)\right\rangle,
\end{aligned}
$$

where the vector and colour indices have been suppressed.

We do not consider the path $C$ to be extended to complex values of the time $t$, nor to be closed. Moreover, to some extent we take a step backwards by restoring many elements of the old-fashioned perturbation theory which deals with retarded and advanced propagators, and incorporates microcausality in the sense of Yang-Feldman equations 汪. The $C$-contour technique is only a convenient trick to do this in an economic way. Similarly, the $T$-ordered Green function is the sole carrier of physical information only under very special circumstances. Indeed, by definition

$$
\mathbf{G}_{00}(x, y)=-i \operatorname{Tr}\left[\rho S^{\dagger} T(q(x) \bar{q}(y) S)\right] .
$$

If the density matrix of the system, $\rho$, corresponds to the exact stationary ground state, then in presence of an interaction the state-vector can acquire only a phase factor, and we obtain Feynman's Green function:

$$
\mathbf{G}_{F}(x, y)=\frac{-i \operatorname{Tr} \rho T(q(x) \bar{q}(y) S)}{\langle S\rangle_{0}}
$$

As we shall see in a while, the Green functions $\mathbf{G}_{00}(x, y)$ and $\mathbf{G}_{F}(x, y)$ even obey different integral equations. 
The choice of the density matrix specifies the physical phenomenon under consideration. If the initial state of the system consists of a only few excitations of the vacuum, then we have the density matrix of a pure state. In this case we are dealing with the well known picture of scattering. Eventually, this situation is described by a certain set of vacuum expectation values, and the corresponding density matrix of the pure ground state is

$$
\rho_{Q C D}=\left|0_{Q C D}\right\rangle\left\langle 0_{Q C D}\right| .
$$

The various density matrices which we shall use later for the computation of quark and gluon production in the first hard AA-collision, and for the rates of the photon and dilepton emission from the QGP, emerge from the following scenario for the heavy ion collision: In the initial stages of a collision at RHIC or LHC energies $(\tau \sim 0.1 \mathrm{fm} / \mathrm{c})$, hard impacts take place. The initial state that precedes these processes consists of two stable, well shaped nuclei moving along opposite directions of the light cone, and the density matrix for each of them is the same as in the deep inelastic e-p or $\mu \mathrm{A}$ scattering. At this stage, the most interesting observables are the quark and gluon distributions after the first interaction that destroyed the nucleons. The QGP domain begins somewhat later when the distributions are already chaotized, and may be described by one-particle distributions. The most general density matrix which simulates any given form of a one-particle distribution is of the following form:

$$
\rho=\prod_{N} \prod_{p, j} e^{-f_{j}(N, p) a_{j}^{\dagger}(N, p) a_{j}(N, p)},
$$

where $N$ labels the space cells at the hypersurface of the initial data, and $n_{j}(N, p)=a_{j}^{\dagger}(N, p) a_{j}(N, p)$ is an operator of the number of partons of type $j$ and quantum number $p$ in the $N$-th cell. Thus, we completely neglect all correlation effects in the initial phase space. Introduction of the cells is necessary only for extended objects without long-scale quantum coherence. For a single hadron they are not needed.

The density matrix of the Gibbs ensemble of noninteracting quarks and gluons against a hydrodynamic background is of the same kind as (3.10), and obeys the additional condition of being an entropy extremum. Introducing the local 4 -velocity of continuous media $u^{\mu}(x)$ we may write

$$
\rho_{Q C D}=\prod_{N} \frac{\exp \left[\left(-P_{N} u_{N}+\mu_{N} Q_{N}\right) / T_{N}\right]}{S p\left[\exp \left[\left(-P_{N} u_{N}+\mu_{N} Q_{N}\right) / T_{N}\right]\right]}
$$

where $P_{N}^{\mu}$ and $Q_{N}$ are the total 4-momentum and (baryonic) charge of free quarks and gluons at temperature $T_{N}$, and $\mu_{N}$ is the chemical potential in a small 3-volume $V_{N}$ on the hypersurface of the initial data.

The explicit form of the "bare" Greenians which are the basis of the perturbation theory is quite evident: The "vacuum" Green functions are of the standard form,

$$
\begin{aligned}
& G_{10,01}^{(0)}(s)=-2 \pi i(\beta+m) \theta\left( \pm s_{0}\right) \delta\left(s^{2}-m^{2}\right), \quad G_{00,11}^{(0)}(s)= \pm \frac{\not s+m}{s^{2}-m^{2} \pm i 0} \\
& D_{10,01}^{(0) \mu \nu}(s)=\quad-2 \pi i d^{\mu \nu}(s) \theta\left( \pm s_{0}\right) \delta\left(s^{2}\right), D_{00,11}^{(0) \mu \nu}(s)=\quad \pm \frac{d^{\mu \nu}(s)}{s^{2} \pm i 0} .
\end{aligned}
$$

where the projector $d^{\mu \nu}(s)$ depends upon the choice of gauge. Usually, the bare Greenians of the ensemble are of the form

$$
G_{A B}(s)=G_{A B}^{(0)}(s)+G_{\beta}(s), \quad D_{A B}^{\mu \nu}(s)=D_{A B}^{(0) \mu \nu}(s)+D_{\beta}^{\mu \nu}(s),
$$

where the additional terms originating from the $\rho_{i n}$,

$$
\begin{array}{r}
G_{\beta}(s)=2 \pi i(\beta+m) \delta\left(s^{2}-m^{2}\right)\left[\theta\left(s_{0}\right) n^{(+)}(s)+\theta\left(-s_{0}\right) n^{(-)}(s)\right], \\
D_{\beta}^{\mu \nu}(s)=-2 \pi i d^{\mu \nu}(s) \delta\left(s^{2}\right)\left[\theta\left(s_{0}\right) f^{(+)}(s)+\theta\left(-s_{0}\right) f^{(-)}(s)\right],
\end{array}
$$

manifestly contain the Fermi- or Bose-occupation numbers, $n^{( \pm)}$or $f^{( \pm)}$, respectively. They are the diagonal elements of the density matrix. All theorems of the Wick-type, which are necessary for the calculations, can be proven easily for density matrices like (3.10) and (3.11).

\subsection{The Schwinger-Dyson equations for $Q F K$}


Except for the matrix form, the Schwinger-Dyson equations for the Heisenberg correlators remain the same as in any other technique. An elegant and universal way to derive them which does not rely on the initial diagram expansion can be found in Ref. [2]. For the quark field these equations are of the form

$$
\mathbf{G}_{A B}=G_{A B}+\sum_{R S} G_{A R} \circ \Sigma_{R S} \circ \mathbf{G}_{S B},
$$

Here the dot stands for convolution in coordinate space, and for the usual product in momentum space (providing the system can be treated as homogeneous in space and time). Indeed, the only tool used to derive these equations was the Wick theorem for the ordered products of the operators. The type of ordering is inessential [2]. Explicit expressions for the self-energies are obtained automatically in course of the derivation of the Schwinger-Dyson equations. The quark self-energy matrix reads

$$
\Sigma_{A B}(x, y)=i(-1)^{A+B} g_{r}^{2} \sum_{R, S=0}^{1}(-1)^{R+S} \int d \xi d \eta t^{a} \gamma^{\mu} \mathbf{G}_{A R}(x, \xi) \Gamma_{R B, S}^{d, \lambda}(\xi, y ; \eta) \mathbf{D}_{S A, \lambda \mu}^{d a}(\eta, x) .
$$

The form the strong $q q B$-vertex that appears is

$$
\Gamma_{S Q, P}^{d, \lambda}(x, y ; z)=(-1)^{P+S+Q} \frac{\delta\left[\mathbf{G}^{-1}(x, y)\right]_{S Q}}{g_{0} \delta \mathcal{B}_{\lambda}^{d}\left(z_{P}\right)},
$$

which is the functional derivative of the inverse Greenian of the quark field with respect to the "external" gluon field $\mathcal{B}(x)$.

The matrix of the photon correlators obeys similar equations,

$$
\boldsymbol{\Delta}_{A B}=\Delta_{A B}+\sum_{R S} \Delta_{A R} \circ \Pi_{R S} \circ \boldsymbol{\Delta}_{S B},
$$

where the electromagnetic polarization operator is

$$
\mathcal{P}_{A B}^{\mu \nu}(x, y)=-i(-1)^{A+B} g_{r}^{2} \sum_{R, S=0}^{1}(-1)^{R+S} \int d \xi d \eta \gamma^{\mu} \mathbf{G}_{A R}(x, \xi) E_{R S, B}^{\nu}(\xi, \eta ; y) \mathbf{G}_{S A}(\eta, x),
$$

and where the electromagnetic vertex

$$
E_{R S, P}^{\lambda}(x, y ; z)=(-1)^{R+S+P} \frac{\delta\left[\mathbf{G}^{-1}(x, y)\right]_{R S}}{e \delta \mathcal{A}_{\lambda}\left(z_{P}\right)}
$$

is dressed by the strong interaction. The latter in turn obeys the equation

$$
E^{\mu}=\gamma^{\mu}+E^{\mu} \circ \mathbf{G G} \circ \mathbf{K}
$$

with a four-fermion vertex $\mathbf{K}$.

The gluon field correlators also obey similar equations

$$
\mathbf{D}_{A B}=D_{A B}+\sum_{R S} D_{A R} \circ \Pi_{R S} \circ \mathbf{D}_{S B},
$$

where the gluon self-energy has two terms:

$$
\begin{aligned}
\Pi_{A B}^{\mu \nu, a b}(x, y)=- & i g_{r}^{2} \sum_{R, S=0}^{1}(-1)^{A+B+R+S}\left[\int d \xi d \eta \operatorname{Tr} \gamma^{\mu} \mathbf{t}^{a} \mathbf{G}_{A R}(x, \xi) \Gamma_{R S, B}^{\nu, b}(\xi, \eta ; y) \mathbf{G}_{S A}(\eta, x)-\right. \\
& \left.-\int d \xi d \eta V_{a c f}^{\mu \alpha \lambda}\left(x, \xi \eta^{\prime}\right) \mathbf{D}_{A R}^{c c^{\prime}, \alpha \beta}\left(\xi, \xi^{\prime}\right) \mathbf{V}_{R S B ; b c^{\prime} f^{\prime}}^{\nu \beta \sigma}\left(\xi^{\prime}, \eta, y\right) \mathbf{D}_{S A}^{f^{\prime} f, \lambda \sigma}\left(\eta, \eta^{\prime}\right)\right]
\end{aligned}
$$

The 3-gluon vertex (in coordinate representation) is defined as

$$
\mathbf{V}_{b c f, R S P}^{\nu \beta \sigma}(x, y, z)=(-1)^{R+S+P} \frac{\delta\left[\mathbf{D}^{-1}(x, y)\right]_{R S}^{b c ; \nu \beta}}{g_{r} \delta \mathcal{B}_{\sigma}^{f}\left(z_{P}\right)}
$$


We have omitted the trivial term with the 4-gluon vertex in Eq. (3.23). The explicit coordinate expression for the bare 3 -gluon vertex is

$$
V_{b c f, R S P}^{\nu \beta \sigma}(x, y, z)=(-1)^{R+S+P} \frac{\delta\left[D_{(0)}^{-1}(x, y)\right]_{R S}^{b c ; \nu \beta}}{g_{r} \delta \mathcal{B}_{\sigma}^{f}\left(z_{P}\right)},
$$

where the inverse Green function $D_{(0)}^{-1}$ denotes a linearized differential operator of the wave equation for the gluon in an external gluon field. The only importance of 3-gluon vertex in this form is that it displays its local nature, $V_{A B C}(x, y, z) \sim \delta_{A B} \delta_{A C} \delta(x-y) \delta(x-z)$. In momentum representation it may be written as

$$
V_{A B C ; a b c}^{\alpha \beta \gamma}\left(p_{1}, p_{2}, p_{3}\right)=-i g_{r} \delta_{A B} \delta_{A C} f^{a b c}\left[g^{\alpha \beta}\left(p_{1}-p_{2}\right)^{\gamma}+g^{\beta \gamma}\left(p_{2}-p_{3}\right)^{\alpha}+g^{\gamma \alpha}\left(p_{3}-p_{1}\right)^{\beta}\right] \quad .
$$

The four types of operator ordering which enter Eqs. (3.1) are not linearly independent, i.e., there exists a set of relations between the field correlators, and between the self-energies:

$$
\begin{gathered}
G_{00}+G_{11}=G_{10}+G_{01}, \quad \Sigma_{00}+\Sigma_{11}=-\Sigma_{10}-\Sigma_{01}, \\
D_{00}+D_{11}=D_{10}+D_{01}, \quad \Pi_{00}+\Pi_{11}=-\Pi_{10}-\Pi_{01} .
\end{gathered}
$$

These indicate that only three elements of the $2 \times 2$ matrices $G, \Sigma$, etc. are independent. To remove the overdetermination let us introduce new functions

$$
\begin{array}{ccc}
G_{r e t}=G_{00}-G_{01}, & G_{a d v}=G_{00}-G_{10}, & G_{1}=G_{00}+G_{11} ; \\
\Sigma_{r e t}=\Sigma_{00}+\Sigma_{01}, & \Sigma_{a d v}=\Sigma_{00}+\Sigma_{10}, & \Sigma_{1}=\Sigma_{00}+\Sigma_{11},
\end{array}
$$

as well as their analogs for bosonic correlators. One of the possible ways to exclude the extraneous quantities is to use the following unitary transformation [1],

$$
\tilde{G}=R^{-1} G R, \quad \tilde{\Sigma}=R^{-1} \Sigma R, \quad R=\frac{1}{\sqrt{2}}\left|\begin{array}{rr}
1 & 1 \\
-1 & 1
\end{array}\right| .
$$

In this new representation, the matrices of the field correlators and self-energies have a triangle form,

$$
\tilde{G}=\left|\begin{array}{ll}
0 & G_{a d v} \\
G_{r e t} & G_{1}
\end{array}\right|, \quad \tilde{M}=\left|\begin{array}{ll}
\Sigma_{1} & \Sigma_{r e t} \\
\Sigma_{a d v} & 0
\end{array}\right|
$$

Applying transformation (3.29) to the matrix Schwinger-Dyson equations (3.15) we may rewrite them in the following form:

$$
\begin{gathered}
\mathbf{G}_{r e t}=G_{r e t}+G_{r e t} \circ \Sigma_{r e t} \circ \mathbf{G}_{r e t}, \\
\mathbf{G}_{a d v}=G_{a d v}+G_{a d v} \circ \Sigma_{a d v} \circ \mathbf{G}_{a d v}, \\
\mathbf{G}_{1}=G_{1}+G_{r e t} \circ \Sigma_{r e t} \circ \mathbf{G}_{1}+G_{1} \circ \Sigma_{a d v} \circ \mathbf{G}_{a d v}+G_{r e t} \circ \Sigma_{1} \circ \mathbf{G}_{a d v}
\end{gathered}
$$

There are now two ways to proceed with a further study of Eq. (3.33). If, as typical of condensed matter systems, one has two well separated short- and long-range scales, then it is reasonable to look for a kind of differential kinetic equation [1]. The time- and space-derivatives on its l.h.s. correspond to the slow variations of collective parameters, while the short-distance dynamics is absorbed into the collision term. The existence of two separate scales here is considered part of the external physical input. It must be either self-evident, or be proven by a separate study (confirmed by observations). In order to derive the quasi-classical kinetic equation, one acts on Eq. (3.33) separately from the left and from the right with the differential operator of the free wave equation, and takes the difference of the two resulting equations. Further, it is useful to transform $\mathbf{G}_{1}$ to Wigner variables, which effectively separate the short- and long-range scales. This procedure results in an integro-differential kinetic equation for the density $\mathbf{G}_{1}$ in phase space. It is differential with respect to the long scale, and integral with respect to the short scale.

In relativistic Boltzmann-type kinetic theories one encounters specific difficulties: First, the phase-space Wigner distributions are no longer positive-definite, and thus do not carry any direct physical information. Second, for phenomena like heavy ion collisions, the two scale dynamics is not evident a priori. Thus, it is safer to accept the 
integral equations (3.31)-(3.33) as they are; however, in this form they are badly suited for practical calculations. In a short while we shall show that the exact equations (3.31)-(3.33) can be solved (at least formally).

\subsection{The formal solution of the integral equations}

The solution we shall look for now is equivalent to the rearrangement of the perturbation series for observables. It is useful when specific features of the non-equilibrium system must be taken into account. First, let us introduce two new correlators:

$$
G_{0}=G_{r e t}-G_{a d v}=G_{10}-G_{01}
$$

which coincide with the anti-commutator of the quark fields and thus disappear outside the light cone, and

$$
\Sigma_{0}=\Sigma_{r e t}-\Sigma_{a d v}=-\Sigma_{10}-\Sigma_{01}
$$

which is the commutator of two fermion sources and has the same causal properties as (3.34). The integral equation for $G_{0}$ may be derived by taking difference of Eqs. (3.31) and (3.33):

$$
\mathbf{G}_{0}=G_{0}+G_{r e t} \circ \Sigma_{r e t} \circ \mathbf{G}_{0}+G_{0} \circ \Sigma_{a d v} \circ \mathbf{G}_{a d v}+G_{r e t} \circ \Sigma_{0} \circ \mathbf{G}_{a d v} .
$$

The sum and the difference of Eqs. (3.33) and (3.36) give corresponding equations for the off-diagonal correlators $G_{10}$ and $G_{01}$ :

$$
\mathbf{G}_{01}=G_{10}+G_{r e t} \circ \Sigma_{r e t} \circ \mathbf{G}_{01,10}+G_{\substack{10 \\ 10}} \circ \Sigma_{a d v} \circ \mathbf{G}_{a d v}-G_{r e t} \circ \Sigma_{01} \circ \mathbf{G}_{a d v} .
$$

Since Eq. (3.31) for the retarded propagator may be identically rewritten in the same form,

$$
\mathbf{G}_{r e t}=G_{r e t}+G_{r e t} \circ \Sigma_{a d v} \circ \mathbf{G}_{a d v}+G_{r e t} \circ \Sigma_{r e t} \circ \mathbf{G}_{r e t}-G_{r e t} \circ \Sigma_{a d v} \circ \mathbf{G}_{a d v},
$$

we may use Eq. (3.37) and derive the corresponding equations for the $T$ - and $T^{\dagger}$-ordered propagators:

$$
\mathbf{G}_{00}=G_{11}+G_{r e t} \circ \Sigma_{r e t} \circ \mathbf{G}_{11}+G_{11} \circ \Sigma_{a d v} \circ \mathbf{G}_{a d v}+G_{r e t} \circ \sum_{00} \circ \mathbf{G}_{a d v} .
$$

This chain of routine transformations reduces equations for all elements of the matrix correlator $G_{A B}$ to a unified form. On the one hand, this representation shows that linear relations between the correlators (or, explicitly, different types of orderings) hold even for the equations that the correlators obey. On the other hand, this representation of the equations singles out the role of retarded and advanced propagators over all other correlators. In order to understand

why their role is special, let us transform them further, and begin by rewriting of Eq. (3.33) for the density of states $\mathbf{G}_{1}$ identically as:

$$
\left(1-G_{r e t} \circ \Sigma_{r e t}\right) \circ \mathbf{G}_{1}=G_{1} \circ\left(1+\Sigma_{a d v} \circ \mathbf{G}_{a d v}\right)+G_{r e t} \circ \Sigma_{1} \circ \mathbf{G}_{a d v} \quad .
$$

Since $\mathbf{G}_{r e t} \circ \Sigma_{r e t} \circ G_{r e t}=\mathbf{G}_{r e t}-G_{r e t}$, it is easy to show that

$$
\left(1+\mathbf{G}_{r e t} \circ \Sigma_{r e t}\right)\left(1-G_{r e t} \circ \Sigma_{r e t}\right)=1 .
$$

Further, we have the following two relations

$$
\left(1+\Sigma_{a d v} \circ \mathbf{G}_{a d v}\right)=G_{(0)}^{-1} \circ \mathbf{G}_{a d v}, \quad\left(1+\mathbf{G}_{r e t} \circ \Sigma_{r e t}\right)=\mathbf{G}_{r e t} \circ \stackrel{\leftarrow}{-1}
$$

where

$$
G_{(0)}^{-1}(x)=i \not \partial \partial_{x}-m, \quad \overleftarrow{G_{(0)}^{-1}}(x)=-i \not \partial_{x}-m,
$$

are the left and right differential operators of the Dirac equation, respectively. Now, multiplying Eq. (3.40) by $\left(1+\mathbf{G}_{r e t} \circ \Sigma_{r e t}\right)$ from the left, we find the final form of the equation we are looking for:

$$
\mathbf{G}_{1}=\mathbf{G}_{r e t} \circ \stackrel{\leftarrow}{G_{(0)}^{-1}} \circ G_{1} \circ G_{(0)}^{-1} \circ \mathbf{G}_{a d v}+\mathbf{G}_{r e t} \circ \Sigma_{1} \circ \mathbf{G}_{a d v}
$$


Repeating these transformations for the other equations, we obtain the corresponding forms that are most convenient for subsequent analysis:

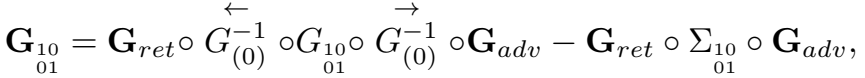

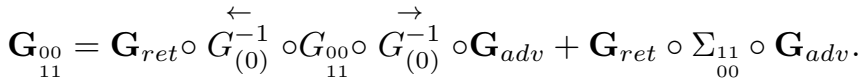

While equations (3.37) and (3.39) are standard integral equations which have the unknown function on both sides, after transformation the unknown function appears only on the 1.h.s., and we may consider Eqs. (3.44)-(3.46) as the formal representation of the required solution.

At this point, the first and the most naive idea is to ignore the arrows indicating the direction the differential operators act in, and to rewrite Eqs. (3.45)-(3.46) in momentum representation. Then the first term in each of Eqs. 3.45) will contain the expression $\left(p^{2}-m^{2}\right) \delta\left(p^{2}-m^{2}\right)$, which equals zero. It reflects simple fact that the offdiagonal correlators $G_{10}$ and $G_{01}$ are solutions of the homogeneous Dirac equation. However, this approach does not appear to be sufficiently consistent: we would lose the identity between Eqs. (3.45) and (3.37), and make it impossible to generate the standard perturbative expansion in powers of the coupling constant. Eqs. (3.46) will be corrupted also.

A more careful examination of Eqs. (3.45)-(3.46) shows that all four dressed correlators $\mathbf{G}_{A B}$ can be found as the formal solution of the retarded Cauchy problem, with bare Greenians as initial data and the self-energies as the sources. Indeed, integrating the first term of each these equations twice by parts, we find for all four elements of $\mathbf{G}_{A B}$

$$
\begin{aligned}
& \mathbf{G}_{01}(x, y)=\int d \Sigma_{\mu}^{(\xi)} d \Sigma_{\nu}^{(\eta)} \mathbf{G}_{r e t}(x, \xi) \gamma^{\mu} G_{01}(\xi . \eta) \gamma^{\nu} \mathbf{G}_{a d v}(\eta, y)-\int d^{4} \xi d^{4} \eta \mathbf{G}_{r e t}(x, \xi) \Sigma_{01}(\xi . \eta) \mathbf{G}_{a d v}(\eta, y)
\end{aligned}
$$

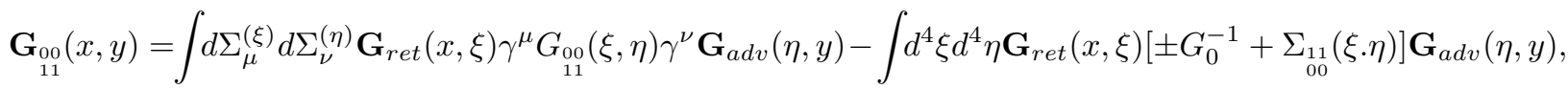

At first glance it is ambiguous to use the local differential operator $G_{0}^{-1}$ in Eq.(3.48) without indication of direction it acts. Nevertheless, as follows from (3.42,

$$
\begin{array}{r}
\mathbf{G}_{r e t} \circ G_{(0)}^{-1} \circ \mathbf{G}_{a d v}-\mathbf{G}_{r e t} \circ G_{(0)}^{\leftarrow} \circ \mathbf{G}_{a d v}=\left(1+\mathbf{G}_{r e t} \circ \Sigma_{r e t}\right) \circ \mathbf{G}_{a d v}-\mathbf{G}_{r e t} \circ\left(1+\Sigma_{a d v} \circ \mathbf{G}_{a d v}\right)= \\
=-\mathbf{G}_{0}+\mathbf{G}_{r e t} \circ \Sigma_{0} \circ \mathbf{G}_{a d v}=0,
\end{array}
$$

and both directions lead to the same answer.

The corresponding equations for boson fields are obtained by replacing $\mathbf{G}_{A B} \rightarrow \mathbf{D}_{A B}$ and $\mathbf{M}_{A B} \rightarrow \Pi_{A B}$, and $\gamma^{\mu} d \Sigma_{\mu} \rightarrow \stackrel{\leftrightarrow}{\partial}^{\mu} d \Sigma_{\mu}$ in Eqs. (3.34) through (3.48).

The equations (3.47) and (3.48) as well as their copies for boson fields are the basic equations of relativistic quantum field kinetics. They are identical to the initial system of Schwinger-Dyson equations, but have the advantage that the time direction is explicitly singled out. There are two terms of different origin that contribute to any correlator (and, consequently, to any observable). The first term retains some memory about the initial data. The length of time for which this memory is kept depends on the retarded and advanced propagators. The second term describes the current dynamics of the system. A comparison of these two contribution allows one to judge if the system indeed has two scales.

\section{Perturbation theory for quantum field kinetics}

Any reasonable diagram technique should allow one to assemble certain subsequences of the bare diagrams into larger pieces, viz., the irreducible elements of the skeleton diagrams, such as exact Greenians, self-energies and vertices. The initial standard form of the Schwinger-Dyson equations (3.15), (3.16) and (3.22), (3.23) is an example of such selective summation. To derive these equations we have employed the functional approach, but any dressed field correlator, self-energy or vertex contains the infinite series of its perturbative expansion in powers of the coupling constant. We can reconstruct the formal perturbation series starting from the skeleton form of the exact SchwingerDyson equations. However, it is not expedient to proceed formally and we shall not attempt it here. For example, an explicit series of radiative corrections to the free asymptotic propagation is never retained in calculations. In the 
course of renormalization this series is absorbed into the physical mass of the field. The theory of the deep inelastic e-p scattering that is based on the operator product expansion (OPE) selectively sums perturbation series in order to emphasize the dominance of light cone dynamics, and introduces its own irreducible elements, the structure functions. Just as the masses of free particles, they are taken from the data, and one should not try to obtain them directly from a perturbative expansion. Thus a physical (versus mathematical) study begins when we make the decision on how to sum the perturbation series or, equivalently, what skeleton elements are not to be expanded in a series. The final choice is the matter of taste.

We wish now to perform the perturbative expansion of the skeleton equations (3.15), (3.18) and (3.22). Their matrix form accounts for a possible instability of the initial ground state with respect to interaction, but their mathematical form is precisely the same as in standard field theory where the initial ensemble corresponds to a pure and stable ground state. Any other structure would be surprising, as only the ordering of the operators, regardless of its type, is important. The presence of additional indices does not change the topology of the diagrams.

Eqs. (3.15), (3.18) and (3.22), supplemented by the expressions for the self-energies, can be iterated in a standard way, giving rise to a formal perturbation series in the powers of coupling constant. Graphically, the series reproduces the Feynman diagram expansion with only one difference: each vertex acquires additional index. This kind of expansion proves useful when we solve local problems, such as photon or dilepton emission from a system with slowly varying macroscopic parameters. These parameters are built into the definition of the density matrix and should not change over the time of emission.

We note, however, that this direct expansion is not most rewarding when the composite system undergoes a strong and short impact. Its parameters will change dramatically within a very short time, and the memory about the density matrix of the true initial state is obviously lost. It is then profitable to rearrange Eqs. (3.18) and (3.22) (for example, "rotating" the basis of correlators, as in Eq. (3.30)) in order to emphasize other details of the dynamical process. Since we are interested in processes on the light cone, the leading singularity of the retarded propagators is the main element which should be preserved in course of renormalization.

\subsection{Perturbation theory for the local emission problem}

The quantity which we shall choose to calculate in this section is the observable rate of the electromagnetic emission (real $\gamma$ or virtual $\gamma^{*}$ ). It is expressed via the tensor of the electromagnetic polarization $\mathcal{P}_{10}^{\mu \nu}(-k)$ of the QGP, given by the Eq. (3.19). Let us expand it in a perturbation series up to order $\alpha_{s}$. The polarization tensor $\mathcal{P}^{\mu \nu}$ contains three irreducible elements: two quark correlators and the electromagnetic vertex dressed by the strong interaction. They are given via their functional definitions, and obey the inhomogeneous integral equations (3.15) $-(3.21)$. The free terms of these equations correspond to the bare quark correlators, averaged over the ensemble represented by the density matrix (of non-interacting particles as a first approximation). The density matrix introduces occupation numbers which define the weights of initial and final states for elementary processes.

The Born term emerges when all the irreducible elements are considered as bare:

$$
\mathcal{P}_{\text {Born }}^{\mu \nu}(-k)=i e^{2} N_{c} \int \frac{d^{4} p}{(2 \pi)^{4}} \gamma^{\mu} G_{10}(p-k) \gamma^{\nu} G_{01}(p) .
$$

To obtain corrections of order $\alpha_{s}$ to the electromagnetic polarization, one should begin by iterating Eqs. (3.15) $-(3.21)$ to the same order. This iteration assumes that we write down equations (3.15) for the correlators with the bare Greenians and with self-energies computed to the first non-vanishing order. The vertex is considered to be bare. As a result of these approximations, we obtain the first group of radiative corrections. A second group appears if we keep field correlators bare, but include the first order corrections to the vertex. Eventually, such an expansion results in the following expression:

$$
\begin{aligned}
& \mathcal{P}_{10}^{\mu \nu}(-k)=i e^{2} N_{c} \int \frac{d^{4} p}{(2 \pi)^{4}} \gamma^{\mu} \mathbf{G}_{10}(p-k) \gamma^{\nu} \mathbf{G}_{01}(p)+e^{2} g^{2} N_{c} C_{F} \sum_{R, S=0}^{1}(-1)^{R+S} \times \\
& \times \int \frac{d^{4} p d^{4} q}{(2 \pi)^{8}} D_{S R}(q) \gamma^{\mu} G_{1 R}(p-k) \gamma^{\lambda} G_{R 0}(p+q-k) \gamma^{\nu} G_{0 S}(p+q) \gamma_{\lambda} G_{S 1}(p)
\end{aligned}
$$

where the correlators $\mathbf{G}_{10}$ and $\mathbf{G}_{01}$ should be taken in the form

$$
\mathbf{G}_{01,10}=G_{01,10}-G_{r e t} M_{01,10} G_{a d v}+G_{r e t} M_{r e t} G_{01,10}+G_{01,10} M_{a d v} G_{a d v} .
$$

All diagrams contributing to $\mathcal{P}_{10}(-k)$ up to order $\alpha_{s}$ are depicted in Fig.11. The sum of these diagrams is the squared modulus of the coherent sum of amplitudes of the real processes of photon emission with first virtual corrections. These 
amplitudes are given at Fig.2. The first process is the direct annihilation of a bare $q \bar{q}$ pair to a (virtual) photon, the next three diagrams are radiative contributions to the same process. Next, there follow four diagrams of $q \bar{q}-$ annihilation accompanied by gluon emission and absorption, and two diagrams for Compton scattering of a quark (or antiquark) and a gluon with photon emission. The first loop and the last four loops in Fig.1 are due to real processes, while the others are due to vertex and mass corrections. The correlators corresponding to initial or final states are marked by dashed lines. Lines with arrows correspond to retarded propagators, and arrows inside the loops show retarded self-energies.

A complete calculation is a subject of separate paper. Here we shall only outline the main ideas and results. The technique of QFK allows one to perform all calculations without the assumption of thermal equilibrium. Not only can the statistical weights of the initial and final states of the real processes be of arbitrary form (though compatible with slowly varying macroscopic parameters), but we can also find all the virtual corrections consistently with the same quark-gluon background distributions. The general rule is that infrared divergences caused by the integration over the low momenta domain (of the Bloch-Nordsieck type) cancel out between real and virtual processes, despite the nontrivial population of the phase space. It is not even necessary to introduce any artificial intermediate cut-offs. Formally, for any given $\alpha_{s}$-order the collinear divergences survive, exactly because the phase space of the initial and final states is populated, and the balance required by the KLN theorem does not hold. However, the physical origin of these divergences allows one to conclude that they are shielded by the quark and gluon distributions: infinitely long free propagation in the dense system is impossible.

\subsection{Perturbation expansion for light cone processes}

As was already mentioned in Sections 1 and 2, QFK is equally applicable to the description of any inclusive processes, including those dominated by the light cone dynamics. These are deep inelastic e-p scattering, or deep inelastic collisions of two hadrons or nuclei. In this case the inclusive cross-section of DIS is expressed via the same electromagnetic polarization tensor, but the kinematic region is different.

The cross-section for inclusive quark and gluon production, which might initiate evolution towards a QGP, is also expressed via the off-diagonal self-energies of the colliding system. For example, for gluon production, we have

$$
\frac{d N_{g}}{d \mathbf{p}}=\sum_{\lambda, a} S p \rho_{i n} S^{\dagger} c^{\dagger}(\mathbf{p}, \lambda, a) c(\mathbf{p}, \lambda, a) S=\sum_{\lambda, a} \int d^{4} x d^{4} y \frac{e^{-i p(x-y)}}{(2 \pi)^{3} 2 p^{0}} \epsilon_{\mu}^{(\lambda)} \epsilon_{\nu}^{(\lambda)}\left[-i \Pi_{a a}^{01, \mu \nu}(x, y)\right]
$$

where the gluon polarization tensor $\Pi_{01}$ is obtained by averaging with the density matrix of the system of the two colliding nuclei. The latter is the direct product of two independent density matrices for each nucleus. Thus, to first approximation,

$$
\Pi_{01}^{\mu \nu}(p)=i g_{0}^{2}\left\{\int \frac{d^{4} k d^{4} q}{(2 \pi)^{4}} \delta(k+q-p)\left\{V_{a c d}^{\mu \rho \sigma}(k+q,-q,-k) \mathbf{D}_{01, d d^{\prime}}^{(A) \sigma \beta}(k) V_{b c^{\prime} d^{\prime}}^{\nu \lambda \beta}(-k-q, q, k) \mathbf{D}_{10, f^{\prime} f}^{(B) \rho \lambda}(q)\right]+(A \leftrightarrow B)\right\} .
$$

where each of the gluon field correlators is averaged with the density matrix of one nucleus, $A$ or $B$. The precollision dynamics of the field correlators $\mathbf{D}_{01, d d^{\prime}}^{(A) \sigma \beta}(k)$ and $\mathbf{D}_{10, f^{\prime} f}^{(B) \rho \lambda}(q)$ is naturally described by equations similar to (3.46). The conditions for nuclear propagation along the light cone allows us to suggest that the retarded propagators contribute to the process mainly via their light cone singularity, and can be used without radiative corrections as a first approximation. In this case we may use the form

$$
\mathbf{D}_{01,10}^{(J)}=D_{01,10}^{(J) \#}+D_{01,10}^{(J) *}-\mathbf{D}_{r e t}^{(J)} \Pi_{01,10}^{(J)} \mathbf{D}_{a d v}^{(J)}
$$

where $D_{01,10}^{(J) *}$ describes the population of the gluon states used as initial data (at, for example, some scale $Q_{0}^{2}$ ), and $D_{01,10}^{(J) \#}$ is the density of gluon states in the initially unpopulated continuum.

The full description of the problem and the calculations is the subject of separate paper. Only the main ideas and results will be outlined here. For the sake of simplicity, let us consider the case of pure glue-dynamics. In that case, Eq. (4.5) yields the following evolution equation:

$$
\Pi_{01}^{\mu \nu}(p)=-i g_{r}^{2} \int \frac{d^{4} k}{(2 \pi)^{4}} V_{a c f}^{\mu \alpha \nu}(p, k-p, k)\left[D_{r e t}(k) \Pi_{10}(k) D_{a d v}(k)\right]^{\alpha \beta} V_{b c^{\prime} f^{\prime}}^{\nu \beta \sigma}(-p, p-k,-k) D_{10, f^{\prime} f}^{\# \lambda \sigma}(k-p)
$$

If this equation is rewritten in light cone variables, and projected onto the specific measurement of the unpolarized e-p DIS experiment, it reproduces the well known Altarelli-Parisi evolution equation for the gluon structure function. If $w_{1}(p)$ is defined as the scalar coefficient in the decomposition 


$$
\Pi^{\mu \nu}(p)=g^{\mu \nu} w_{1}(p)+\frac{p^{\mu} p^{\nu}}{p^{2}} w_{2}(p)+\ldots
$$

then the gluon structure function of the LLA reads

$$
G\left(x, Q^{2}\right)=G\left(x, Q_{0}^{2}\right)+\frac{V_{l a b} P^{+}}{(2 \pi)^{3}} \int_{Q_{0}^{2}}^{Q^{2}} d p_{t}^{2} \int d p^{-} \frac{i p^{+} w_{1}^{01}(p)}{\left(p^{2}\right)^{2}}
$$

where $G\left(x, Q_{0}^{2}\right)$ is the phenomenologic "initial" distribution at the scale $Q_{0}^{2}$.

Here, the novel point is that equations like (4.7) are of the evolution type, even in their initial coordinate form. Further, they may be derived without reference to any particular process. The equations are of a ladder form, and the ordering of the ladder cells by the Feynman $x$ and virtuality $Q^{2}$ is a consequence of the initial retarded ordering: lower $x$ and higher $Q^{2}$ correspond to later times in the evolution. A parallel study of the two processes, p-e DIS, and p-p or A-A collisions, shows that one may use the new evolution equations to obtain quantities (like $w_{1}^{01}(p)$ ) which are common to these two processes. Further, one avoids intermediate parton language.

\section{Conclusions}

This paper presents a formalism which allows us to solve various problems of the QGP dynamics using the same technique. On the one hand, it allows one to calculate the rates of such processes as photon and dilepton emission, or heavy quarks production in the QGP. On the other hand, it is a good tool for the study of extremely non-equilibrium process, such as the first hard collision of two heavy ions.

From the most general point of view, this technique is capable of replacing the standard Feynman approach, in any situation where the latter is applicable. In QFK, we begin with the density matrix generated by the Fock operators from the pure vacuum state of non-interacting fields. The reader can easily rewrite some chapters from a standard textbook on QED in terms of this approach as an exercise. In some points the new approach is physically more intuitive than the Feynman technique: at the tree level, retarded propagators appear naturally in every place where we usually put them in by hands (following common sense). The cutting rules and unitarity come to be a trivial consequence of the matrix structure: both $S$ and $S^{\dagger}$ contribute to any observable from the very beginning.

For the case of true thermal equilibrium, this kinetic approach also reproduces all that is obtainable from the standard Matsubara formalism. All global relations like the FDT come to be a trivial consequence of thermal equilibrium and the matrix structure of the Schwinger-Dyson equations, but none of global relations are is used in the definition of the observables. For the Gibbs ensemble, QFK describes dynamical fluctuations.

The essential new point is that the theory is not confined to a specific form of the one-particle distribution. The plasma may be far out of thermal and/or chemical equilibrium. Nevertheless we can consider both real and virtual processes in a self-consistent manner. Usually this consistency is lost in computer simulations of the emission from the non-equilibrium plasma [5]: only tree level matrix elements are taken into account. In the next paper we shall give an example: for dilepton emission from a non-equilibrium "plasma," a self-consistent account of the radiative corrections changes the answers from what is obtained using a naive cut-off of the infrared singularities. We have also found a significant difference in the case of an equilibrated plasma.

The QFK approach allows one to formulate new principles for computing the distributions of quarks and gluons created in the first hard interaction of two heavy ions at high energies. It essentially employs an initial resummation of the perturbation series for the probabilities, and allows one to describe two different high energy processes, viz., e-p scattering and nuclear interactions, in the same terms. These processes are considered as two versions of the same phenomenon - deeply inelastic scattering of composite systems. The calculations can be performed without reference to parton phenomenology.

\section{Acknowledgements}

I am indebted to G. Brown and the Nuclear theory group at SUNY at Stony Brook for support during the spring and summer of 1994 when this work was began. I am grateful to L. McLerran, A.H. Mueller, E.Shuryak, J.Smith, A.Vainshtein R.Venugopalan and G. Welke for many stimulating discussions.

A part of this work was supported by the U.S. Department of Energy under Contract No. DE-FG02-94ER40831.

[1] L.V. Keldysh, Sov. Phys. JETP 20 (1964) 1018; E.M. Lifshits, L.P. Pitaevsky, Physical kinetics, Pergamon Press, Oxford, 1981. 
[2] N.N. Bogolyubov, D.V. Shirkov, Introduction to the theory of quantized fields, Interscience, NY,1959

[3] E. Shuryak, Phys. Rev. Lett. 68(1992)3270.

[4] C.N.Yang, D.Feldman, Phys Rev. 79(1950)972.

[5] K. Geiger: Phys. Rev. D46(1992)4965,4986; P. Lichard, Preprint TPI-MINN-92/51T.

FIG. 1. Diagrams for the rate of dilepton production. The dashed line crosses the Greenians representing densities of the initial or final states. Arrows label the retarded and advanced propagators and show the latest time.

FIG. 2. Processes participating in the dilepton production in the $\alpha_{s}$-order. Notation the same as in Fig.1. 
This figure "fig1-1.png" is available in "png" format from: http://arxiv.org/ps/hep-ph/9412362v1 

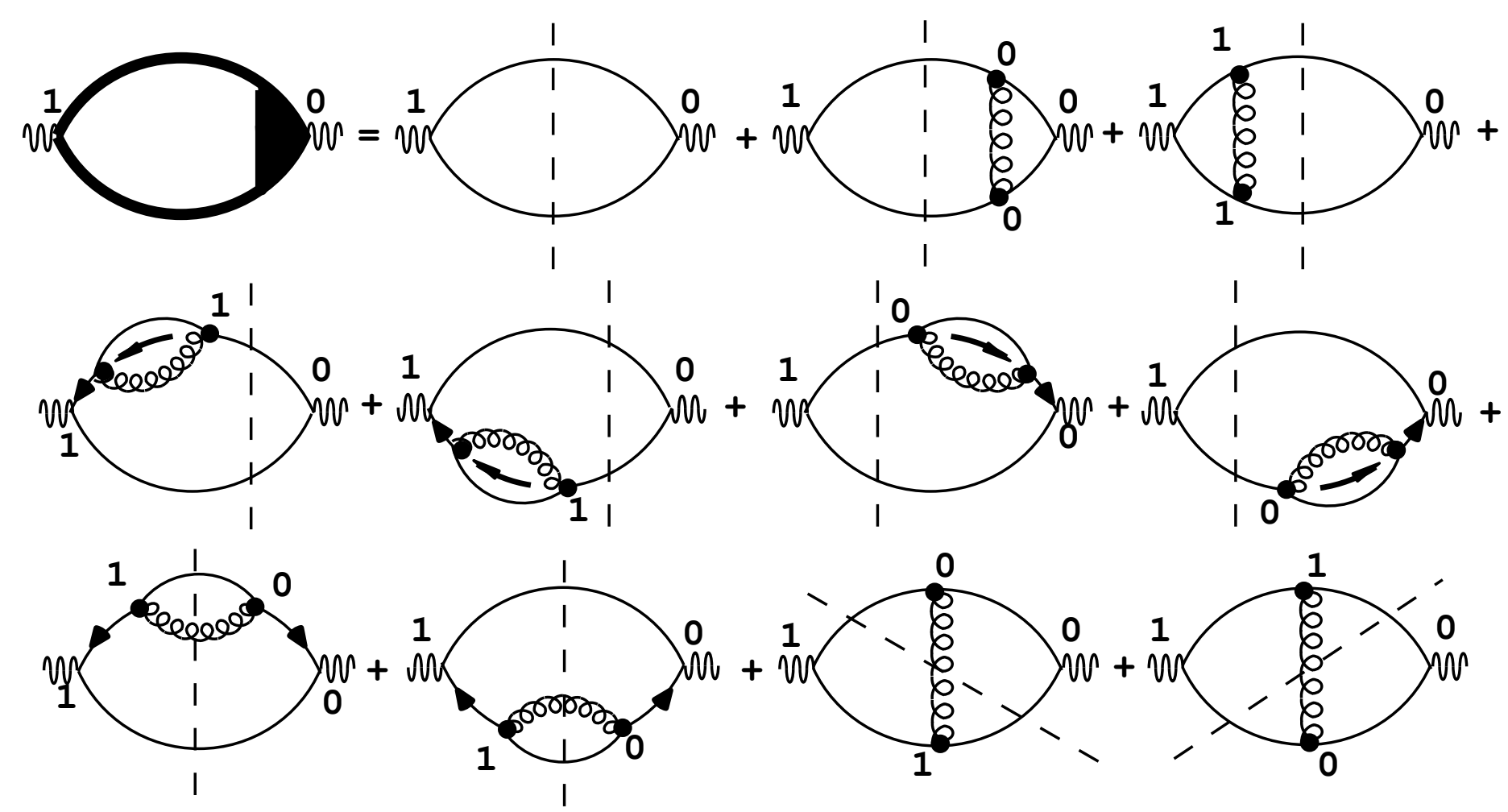

Fig. 1
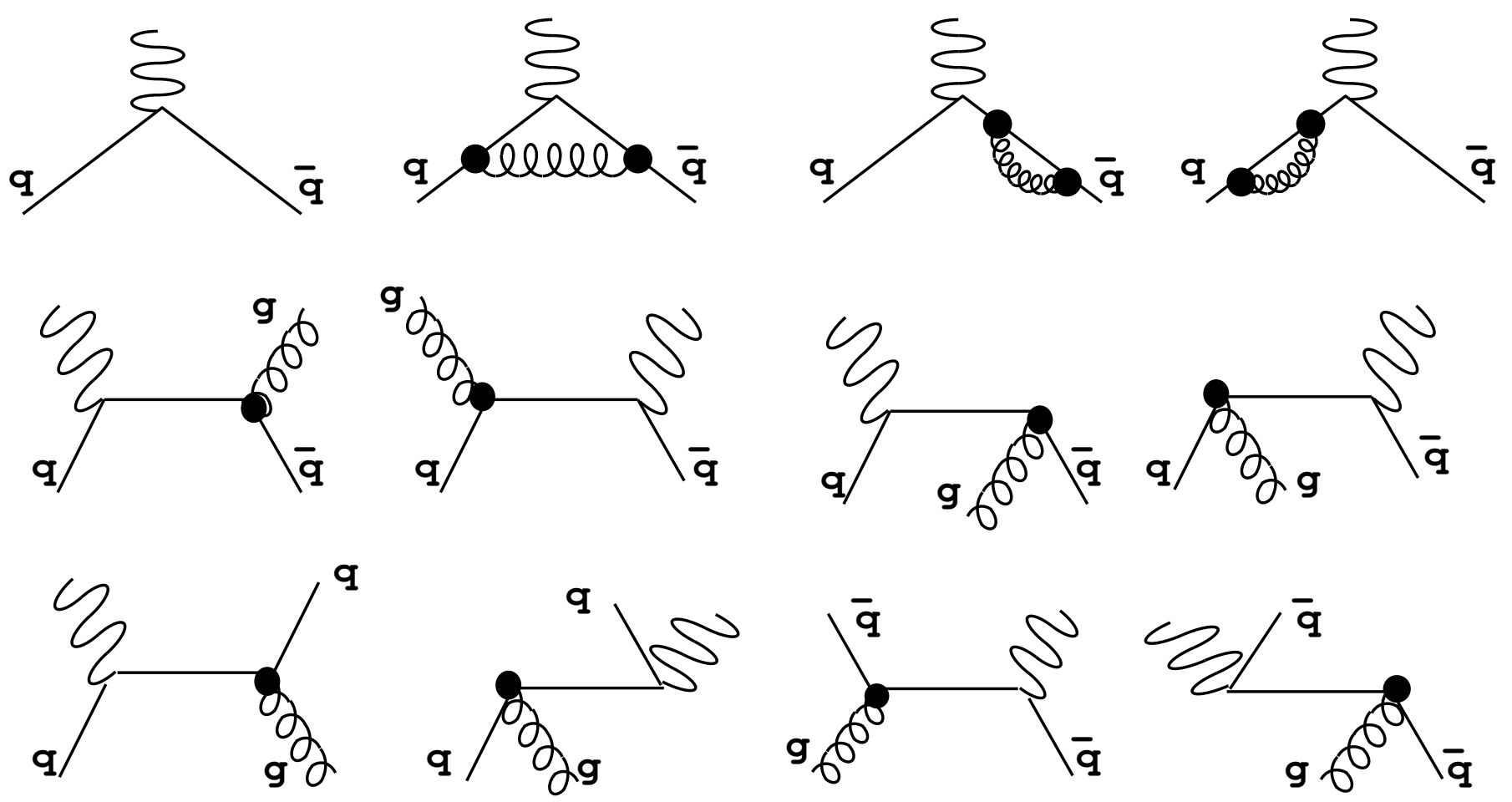

Fig. 2 